\title{
Mathilde Pette
}

Post-doctorante en sociologie

mathilde.pette@gmail.com

\section{Fabien Éloire}

MCF en sociologie

fabien.eloire@univ-lille1.fr

Laboratoire CLERSÉ-CNRS / Université Lille1

\section{Pôles d'organisation et engagement dans l'espace de la cause des étrangers : l'apport de l'analyse des réseaux sociaux.}

Cet article montre dans quelle mesure les analyses de réseaux sociaux (ARS) permettent d'analyser la structure d'un espace militant au regard des pratiques de multi-engagement des militants qui y sont engagés. Les militants sont en effet souvent affiliés à plusieurs organisations (syndicats, partis politiques et associations). L'ARS permet, sur la base de ces données relevant de l'engagement individuel, d'accéder au niveau organisationnel : la coexistence de deux viviers de recrutement des militants, l'un chrétien, l'autre d'extrême gauche à dominante communiste, apparaît ainsi sous un jour complémentaire à travers plusieurs constellations d'associations plus ou moins imperméables entre elles. Cet article montre ainsi comment, en cartographiant l'espace de la cause étudiée, les ARS sont un outil d'analyse pertinent pour saisir les processus d'encastrement organisationnel et de multipositionnalité.

\section{Organizations' poles and commitment in the space of foreigners' defense: the contribution of social network analysis.}

This article shows how social network analysis (SNA) can be used to analyze the structure of an activism space with regard to the multi-commitment practices of the activists who are engaged. Generally activists are affiliated to different organizations (labour unions, political parties and non-profit associations). On the basis of these data related to individual activism, the SNA allows to realize an analysis at the organizational level: thus the coexistence of two recruiting pools for activists, one relating to Christians, the other relating to extreme left, mainly communist, appears to be complementary across several constellations of associations, which are more or less impermeable between themselves. By its way to map the activism space, this article shows how the SNA is a relevant tool for studying organizational embeddedness and multi-positionality processes. 


\title{
Pôles d'organisation et engagement dans l'espace de la cause des étrangers : l'apport de l'analyse des réseaux sociaux.
}

\begin{abstract}
Ce mercredi 23 janvier 2013, l'église Saint Benoît située dans un quartier populaire de Lille est pleine. Plusieurs centaines de personnes sont venues rendre hommage à Gérard Naissant, prêtre, engagé à la Pastorale des migrants, créateur du Groupe de réflexion interreligieux d'aide aux migrants (GRIAM), animateur à Lille des Cercles du Silence et membre de l'association Soutenons, aidons, luttons, agissons pour les migrants et les pays en difficulté (SALAM) à Calais. Beaucoup de présents ont rencontré Gérard en 2007 lors d'une grève de la faim d'étrangers sans-papiers organisée par le Comité des sans-papiers du Nord (CSP59) à la Bourse du Travail de Lille. Depuis le fond de l'église, on voit celles et ceux qui défilent devant le cercueil : une poignée de salariés et bénévoles de la CIMADE, des militants du CSP59, des bénévoles d'associations d'action sociale du quartier et des ex sans-papiers perdus de vue depuis 2007. Il y a aussi quelques militants syndicalistes ainsi que des membres de l'Action catholique ouvrière. Au fond de l'église, un militant anarchiste se tient debout. "Il fallait bien un mec comme ça pour me faire rentrer à nouveau dans une église », confie-t-il. Un passage de l'Évangile de Jésus selon Saint Matthieu est lu : "J'avais faim, et vous m'avez donné à manger ; j'avais soif, et vous m'avez donné à boire ; j'étais un étranger, et vous m'avez accueilli (...) je vous le dis : chaque fois que vous l'avez fait à l'un de ces petits qui sont mes frères, c'est à moi que vous l'avez fait [chapitre 25, 34-40] ». Les regards de nombre de ces militants se croisent, non sans émotion. La petite famille des militants de défense des étrangers semble ce jour-là réunie au-delà de ses divergences.
\end{abstract}

Note d'observation, janvier 2013, Lille.

Cette scène révèle la capacité qu'ont certains événements de forte portée émotionnelle et symbolique, à rassembler des militants aux revendications et modes d'action distincts: certains accompagnent les étrangers dans leurs démarches juridiques et administratives, d'autres choisissent de mener des actions sociales ou d'alphabétisation, d'autres enfin choisissent des actions contestataires (manifestations, occupations ou grèves de la faim par exemple). Plus précisément, elle révèle qu'au-delà de leurs différences manifestes, les associations et mouvements qui interviennent auprès des populations étrangères dans le Nord de la France, font partie d'une même cause et peuvent légitimement faire l'objet d'une analyse conjointe. Cependant, dans la plupart des travaux consacrés au militantisme, les associations et mouvements de défense des étrangers ont été abordés sous forme de monographies, telles que celles concernant l'ASTI (Lechien, 2003), la FASTI et le MRAP (Siméant, 1998a), le RESF (Mathieu, 2010a, 2010b), le mouvement contre la double peine (Mathieu, 2006), ou plus récemment les grèves de travailleurs sans-papiers (Barron et alii, 2011). Les travaux de Liora Israël (2003, 2009), d'Anna Marek (2003) et de Marie-Hélène Lechien (2002) consacrés au GISTI, comme ceux de Jérôme Drahy au sujet de la CIMADE (2004), permettent de saisir comment l'usage du droit dans la cause des étrangers a des effets sur le travail militant et sur les trajectoires des personnes engagées. Les travaux de Nicolas Fischer (2009), eux aussi réalisés à la CIMADE et consacrés à l'accompagnement et à la défense des étrangers retenus dans les centres de rétention administrative, permettent d'étudier les pratiques militantes en situation d'enfermement.

Cet article, qui prend pour objet la cause des étrangers entendue comme l'ensemble des associations et des mouvements qui aident, accueillent et défendent les étrangers présents sur le territoire français, et ceci quelles que soient leurs nationalités et leurs statuts, se place dans la lignée de ces travaux. 
Cependant, il propose d'opérer un double redéploiement du questionnement autour de cet objet: premièrement, il invite à embrasser la cause étudiée dans son ensemble plutôt que de l'évoquer sous l'angle d'une organisation spécifique; deuxièmement, il propose d'analyser conjointement les organisations militantes proprement dites et les positionnements individuels des militants, et ce à travers l'étude de leurs engagements multiples.

Pour cela, il choisit d'envisager simultanément l'ensemble de ces associations et mouvements comme constitutifs d'un même espace militant - l'espace de la « cause des étrangers - lui-même situé au sein d'un espace plus vaste, celui de l'«espace des mouvements sociaux» tel que proposé par Lilian Mathieu $(2007,2012)$. En effet, l'auteur propose, selon nous, un outil stimulant pour penser l'engagement et le militantisme en considérant que « l'activité protestataire relève d'un domaine de pratique et de sens relativement autonome au sein du monde social » et en postulant que cet espace est un univers « doté de logiques, de modes de fonctionnement, d'enjeux et de références propres » (Mathieu, 2012, p. 9). Cette perspective se justifie ici dans la mesure où la cause des étrangers s'est construite historiquement, au cours du XXe siècle, en lien étroit avec la construction de l'immigration comme un nouveau problème politique (Lenoir, 1989 ; Laurens, 2008; Noiriel, 2008), autour de quatre formes d'action distinctes : l'action sociale, l'alphabétisation, la défense des droits et les luttes revendicatives. Si chacune d'entre elles renvoie à des répertoires d'action (Tilly, 1984), des revendications et des filiations historiques distinctes, ces formes d'action coexistent aujourd'hui au sein de l'espace de la cause des étrangers.

Cette perspective d'étude de la cause des étrangers à travers la notion d'espace militant permet aussi d'appréhender l'articulation des logiques organisationnelles et individuelles du militantisme en portant une attention particulière aux pratiques de multi-engagement des militants (Fillieule et alii, 2004). Si cette question du multi-engagement n'est pas nouvelle dans les travaux consacrés à l'engagement et aux mobilisations (Oberschall, 1973, Gaxie, 1977, McAdam, 1988), il s'agit ici de l'interroger en combinant les méthodes qualitatives et quantitatives de l'enquête en sciences sociales, et notamment celle de l'analyse des réseaux sociaux. Le multi-engagement est alors envisagé sous l'angle des pratiques individuelles qui viennent éclairer les liens, les proximités et les distances, entre les organisations militantes de la cause étudiée. Partant de cette idée que les individus et les groupes sont interdépendants, Ronald Breiger (1974) avait proposé une technique pour modéliser cette dualité à travers des représentations matricielles ${ }^{1}$. Celle-ci a, jusqu'à maintenant, surtout été mobilisée dans le cadre de la sociologie des classes dirigeantes et de la sociologie économique, notamment à propos de l'étude des liens dits interlocks entre grandes entreprises ${ }^{2}$ (Mizruchi, 1996 ; Dudouet, Grémont, 2007 ; Finez, Comet, 2011). Cependant, cette technique nous est apparue ici heuristique dans le cadre d'une sociologie de l'engagement et du militantisme ${ }^{3}$ dans la mesure où elle donne à voir simultanément les organisations et leurs militants imbriqués dans des processus d'encastrement organisationnel.

En nous appuyant sur les données recueillies lors de notre enquête doctorale (Encadré 1), nous montrerons que si les militants se caractérisent par une forte homogénéité sociale, l'analyse de réseaux fait cependant apparaître un espace d'organisations structuré, et en son sein deux grands pôles d'engagement qui y coexistent.

\footnotetext{
${ }^{1}$ Il conceptualise le passage d'une matrice 2-mode (en ligne, les individus; en colonne, les organisations), à une matrice 1mode (interpersonnelle ou intergroupe).

${ }^{2}$ Un lien interlock est un lien de coappartenance. Appliqué au monde des grandes entreprises, il signifie que deux entreprises sont liées dès lors qu'elles ont en commun au moins un membre de leur conseil d'administration.

${ }^{3}$ L'analyse de réseaux sociaux est complémentaire de l'analyse des correspondances multiples (ACM). L'ACM permet de mieux cerner les processus d'engagement individuel (au sujet des incidences politiques de la participation à Mai 68, par exemple cf. Pagis, 2011 ; sur notre objet, cf. Pette, 2012). Cependant, alors que les axes de l'ACM se voient déformés par les variables à petits effectifs, l'ARS offre, elle, l'avantage de pouvoir les représenter sans difficulté (sous la forme d'un lien très fin et d'un point de petite taille), sans pour autant biaiser la structure d'ensemble.
} 


\section{Encadré 1 : Méthodologie}

Entre janvier 2009 et juin 2012, nous avons réalisé une enquête de terrain menée dans le Nord de la France auprès d'une population de militants de la cause des étrangers (Pette, 2012). Quatre types de matériaux ont été mobilisés, mêlant approches qualitative et quantitative: des entretiens avec des militants et responsables associatifs $(n=80)$; des observations en situation dans des associations et à la Préfecture du Nord; le dépouillement d'archives du Bureau des associations de la Préfecture du Nord et du quotidien régional La Voix du Nord; et enfin, un questionnaire de 44 questions, administré en autopassation par voie postale et par email ${ }^{4}$, auprès de 389 militants membres d'au moins une des associations que nous avons repérées, durant l'enquête de terrain, comme étant susceptibles de former l'ossature de l'espace militant de la cause des étrangers dans le Nord de la France.

\section{Un même monde social, des militants multi-engagés}

Lorsque l'on compare les caractéristiques sociodémographiques des personnes investies dans la cause des étrangers à celles de la population française (Tableaux 1 et 2), on ne peut que constater la forte homogénéité sociale qui caractérise ce monde militant. Ils sont ou ont été ${ }^{2}$ le plus souvent des cadres et professions intellectuelles supérieures $(17,5 \%$ des actifs et $16,5 \%$ des retraités) ou des professions intermédiaires (respectivement $28,8 \%$ et $11,8 \%$ ) et sont dotés d'un important capital culturel puisque près de 75\% d'entre eux sont titulaires d'un diplôme supérieur au bac, et plus de six sur dix ont un diplôme de 2ème ou 3ème cycle universitaire, alors même que seuls $21,4 \%$ de la population française a un diplôme du supérieur - cycles courts et longs réunis. Ils appartiennent souvent aux secteurs de l'enseignement, de la santé et du travail social et des arts, donc à la classe des professionnels que Florence Passy (1998) qualifie de « spécialistes socioculturels », et à la « main gauche » de l'État, selon l'expression employée par Pierre Bourdieu (1993), qui désigne ainsi les petits fonctionnaires assurant les fonctions « sociales » de l'État tels que les policiers et magistrats subalternes, les assistantes sociales, les éducateurs, les instituteurs et professeurs. Pour ces derniers, l'engagement militant peut être considéré comme un prolongement de leur pratique professionnelle : que ce soit en tant que travailleurs sociaux, enseignants ou militants, ils restent confrontés aux mêmes populations et aux mêmes difficultés pour répondre aux besoins de celles-ci. Ce constat rejoint les observations faites précédemment par Florence Passy (1998) au sujet des mouvements de solidarité, par Johanna Siméant (1998a) quant aux militants du MRAP, par Emmanuel Reynaud (1980) à propos des militants de groupes des années 1970 tels que le MLAC, le GISTI ou le GIP, ou encore par Érik Agrikoliansky (2002) à propos des militants de la LDH.

\footnotetext{
${ }^{4}$ Le taux de réponse obtenu par voie postale s'élève à $62 \%$.

${ }^{5} 34 \%$ des répondants sont retraités, contre $26,5 \%$ de la population nationale (Insee, Enquête emploi, 2009).
} 
Tableau 1 : Structure par sexe, âge et niveau de diplôme

\begin{tabular}{|c|c|c|c|c|}
\hline & France & \multicolumn{3}{|c|}{ Militants } \\
\hline & & & & $\begin{array}{c}\text { Écart à la } \\
\text { moyenne } \\
\text { nationale }\end{array}$ \\
\hline Hommes & $\%$ & Eff & $\%$ & $-7,05$ \\
\hline Femmes & 48,45 & 161 & 41,4 & $+7,05$ \\
\hline $20-29$ ans & 51,55 & 228 & 58,6 & $-1,2$ \\
\hline $30-39$ ans & 17,8 & 64 & 16,6 & $-4,4$ \\
\hline $40-49$ ans & 18,4 & 54 & 14,0 & $-8,8$ \\
\hline $50-59$ ans & 19,7 & 42 & 10,9 & $+2,0$ \\
\hline $60-69$ ans & 18,7 & 80 & 20,7 & $+9,9$ \\
\hline $70-79$ ans & 15 & 96 & 24,9 & $+3,6$ \\
\hline Aucun diplôme, CEP & 9,4 & 50 & 13,0 & $n r$ \\
\hline Brevet des collèges & 26,8 & $n r$ & $n r$ & $n r$ \\
\hline CAP, BEP & 20,4 & 18 & 4,6 & $-15,8$ \\
\hline Bac, brevet professionnel ou & 13,9 & 50 & 12,9 & $-1,0$ \\
\hline équivalent & 9,6 & 49 & 12,6 & $+3,0$ \\
\hline Supérieur court & 9,6 & $n r$ & $+49,6$ \\
\hline Supérieur long & 11,8 & 239 & 61,4 & $n r$ \\
\hline En cours d'études initiales & 10,5 & $n r$ & $n r$ & \\
\hline
\end{tabular}

Sources : colonne « France » données issues du recensement, Insee, estimations de population (résultats provisoires arrêtés fin 2010) ; colonne « Militants » données issues de notre questionnaire.

Tableau 2 : Structure selon la catégorie socioprofessionnelle

\begin{tabular}{|c|c|c|c|c|}
\hline & France & \multicolumn{3}{|c|}{ Militants } \\
\hline & $\%$ & Eff & $\%$ & $\begin{array}{l}\text { Écart à la } \\
\text { moyenne } \\
\text { nationale }\end{array}$ \\
\hline Agriculteurs exploitants & 1,1 & 2 & 0,5 & $-0,6$ \\
\hline Artisans, commerçants et chefs d'entreprise & 3,4 & 3 & 0,8 & $-2,6$ \\
\hline Cadres et professions intellectuelles supérieures & 8,8 & 68 & 17,5 & $+8,7$ \\
\hline $\begin{array}{l}\text { Dont cadres de la fonction publique, } \\
\text { professions intellectuelles et artistiques }\end{array}$ & 3 & 43 & 11,1 & $+8,1$ \\
\hline Professions Intermédiaires & 13,2 & 112 & 28,8 & $+15,6$ \\
\hline $\begin{array}{l}\text { Dont professions intermédiaires de } \\
\text { l'enseignement, de la santé, de la fonction } \\
\text { publique et assimilés }\end{array}$ & 5,6 & 91 & 23,4 & $+17,8$ \\
\hline Employés & 16,4 & 20 & 5,1 & $-11,3$ \\
\hline Ouvriers & 12,8 & 7 & 1,8 & -11 \\
\hline Retraités & 26,5 & 132 & 33,9 & $+7,4$ \\
\hline Dont anciens cadres & 3,1 & 64 & 16,5 & $+13,4$ \\
\hline $\begin{array}{l}\text { Dont anciennes professions } \\
\text { intermédiaires }\end{array}$ & 5,0 & 46 & 11,8 & $+6,8$ \\
\hline Autres personnes sans activité professionnelle & 17,9 & 41 & 10,5 & $-7,4$ \\
\hline
\end{tabular}

Sources : colonne « France » enquête emploi 2009, Insee ; colonne « Militants » données issues de notre questionnaire. 
L'univers militant donne d'autant plus l'image d'un même monde social (Passy, 1998) que ceux qui le composent ont des pratiques d'engagements multiples, la plupart étant en effet affiliés à plusieurs associations et organisations : $48 \%$ des militants que nous avons interrogés sont ou ont été syndiqués ${ }^{6}$, $20 \%$ sont membres d'un parti politique, $75 \%$ d'au moins une autre association ${ }^{7}$ et plus de $90 \%$ d'entre eux font des dons réguliers ${ }^{8}$ à une ou plusieurs associations. Successivement ou simultanément, à l'occasion d'événements et de la défense de causes distinctes, les militants se rencontrent donc dans différentes organisations et mobilisations et s'insèrent ainsi progressivement dans un réseau militant. L'enquête de terrain menée dans les associations et mouvements de la cause des étrangers du Nord de la France, tout comme notre propre pratique militante dans plusieurs mobilisations sociales et associations, nous ont ainsi amené à croiser régulièrement les mêmes personnes sous des «étiquettes» associatives ou organisationnelles distinctes.

Cette question du multi-engagement n'est pas nouvelle en sociologie. Doug McAdam (1988) souligne, à propos des luttes pour les droits civiques des populations noires en 1964 au Mississippi, dans quelle mesure les liens organisationnels autant que les liens personnels ont fonctionné comme supports de la mobilisation du Freedom Summer. Antony Oberschall (1973), à propos du recrutement des militants, qualifie, lui, de « bloc recruitement » les pratiques par lesquelles les organisations politiques cherchent à trouver de nouveaux adhérents dans des réseaux proches, où les individus sont perçus comme davantage susceptibles d'être interpellés par une cause spécifique. Le contexte relationnel opère selon Florence Passy (1998) comme un «support structurel des mouvements sociaux» en apportant les ressources matérielles, symboliques, politiques et humaines nécessaires à l'émergence de nouvelles mobilisations : une fois le militant investi, son insertion dans des réseaux favorise son maintien et son renforcement dans l'engagement car l'exercice même du militantisme incite à participer à de nouvelles organisations et à s'intéresser à de nouvelles causes. C'est ce phénomène que Daniel Gaxie (1977) - à propos de l'engagement partisan - a nommé l' « effet surgénérateur » de l'engagement. Devenir militant et le rester suscite, selon lui, chez les militants, le sentiment d'appartenir à une "grande famille ». Plus récemment, Hélène Combes (2009) s'est appuyée sur l'étude des pratiques de multi-engagement pour reconstituer les réseaux du Parti de la révolution démocratique au Mexique, en montrant les liens qu'il entretient avec des organisations paysannes, étudiantes, syndicales ou indigènes ${ }^{9}$.

Dans la lignée de tels travaux qui s'efforcent de relier réseaux et militantisme, et qui mettent en avant l'importance des pratiques de multi-engagement, nous nous intéressons ici aux logiques qui font que les niveaux individuels et organisationnels de l'engagement s'imbriquent. Pour cela, nous proposons d'interroger les liens entre organisations militantes, non pas au regard des actions menées conjointement ou encore des revendications communes, mais plutôt à travers les pratiques d'engagements multiples de leurs militants.

\footnotetext{
${ }^{6}$ Seuls $7 \%$ des salariés sont syndiqués en France (Insee, Enquête SRCV-SILC, 2010).

${ }^{7}$ Seuls $34 \%$ de la population nationale adhère au moins à une association (Ibid.).

${ }^{8}$ Les statistiques nationales indiquent qu'en 2005, seuls $9 \%$ des ménages déclarent au moins un don au profit d'un organisme d'aide aux personnes en difficultés. Si ces données sous-estiment la réalité de la pratique du don, en excluant les autres types d'associations et en ne prenant en compte que les foyers ayant déclaré ces dons lors de la déclaration de revenus, la différence entre la population nationale et celle des militants n'en reste pas moins significative (Insee-DGI, Enquête Revenus fiscaux, 2005).

9 À travers cette démarche, elle invite à décloisonner l'étude des relations entre partis et mouvements sociaux pour penser l'unité du militantisme à l'échelle des individus.
} 


\section{Une diversité des formes d'action militantes}

C'est d'abord l'action sociale, destinée aux populations immigrées, qui se développe dans l'aprèsguerre $\left(\right.$ Bernardot, 1999, 2008) ${ }^{10}$ : il s'agit de mettre à disposition des immigrés des biens ou services tels que logements fixes, hébergements temporaires, nourriture ou vêtements, par exemple. Plus tard, le souci de promouvoir l'insertion professionnelle puis l'intégration des immigrés et de leurs familles incite les pouvoirs publics et les associations à développer des actions de formation et d'alphabétisation. Au cours des années 1970 et 1980, l'alphabétisation s'autonomise comme une forme d'action spécifique à travers des cours de français dispensés aux personnes étrangères ou immigrées (Engrand, 1973). Parallèlement, les années 1970 sont aussi marquées par les premières mesures de restriction de l'immigration, à commencer par les circulaires Marcellin-Fontanet en 1972. La cause des étrangers est alors marquée par un « tournant de la défense », incarné au niveau national par le GISTI : la défense des droits des étrangers, et les activités juridiques, deviennent dès lors centrales dans la cause des étrangers et le restent jusqu'à présent (Israël, 2003, 2009 ; Marek, 2003). Enfin, les luttes revendicatives constituent la quatrième forme d'action. Elles émergent au cours des années 1970 (Siméant, 1998a) et se structurent au milieu des années 1990, suite aux mobilisations parisiennes dans les églises de Saint-Ambroise et Saint-Bernard, autour d'un nouvel acteur: les collectifs dits "de » sans-papiers. Les luttes revendicatives mobilisent un répertoire d'action distinct des trois autres formes d'action: manifestations, marches, occupations, grèves des travailleurs (Barron et alii, 2011), grèves de la faim (Siméant, 1998b, 2009) ou des loyers (Hmed, 2007) ou concerts de soutien par exemple.

En ce qui concerne les activités militantes envers les étrangers dans le Nord de la France, ce qui a plus particulièrement attiré notre attention, c'est qu'alors que ces quatre formes d'action coexistent aujourd'hui au sein d'un même espace militant, elles s'observent néanmoins rarement au sein d'une même association ou d'un même mouvement. Les organisations, et de ce fait leurs militants, sont ainsi le plus souvent spécialisés autour d'un type spécifique d'intervention auprès des populations étrangères. C'est le cas parmi les associations et mouvements retenus pour l'enquête par questionnaire. AIDA, SALAM, Terre d'Errance Steenvoorde, Le Grand-Mi et le GRIAM sont des associations d'action sociale qui assurent un suivi individuel et administratif des étrangers, distribuent des repas ou des vêtements ; la CLÉ est une association d'alphabétisation qui organise des cours de français individuels ; la CIMADE est une association de défense des droits qui organise surtout des permanences et un suivi juridiques; le CSP59 s'inscrit quant à lui dans les luttes revendicatives et use d'un répertoire d'action structuré autour de manifestations, d'occupations de lieux symboliques ou de grèves de la faim. Nous nous sommes efforcés ici de saisir ensemble ces quatre formes d'action constitutives de l'espace de la cause des étrangers. Et, pour étudier à la fois la structure et les jeux de distance-proximité entre ces organisations, c'est la méthode de l'analyse des réseaux qui nous est apparue comme la plus adéquate.

\section{Un espace militant structuré par les organisations}

Le premier enjeu de notre analyse consiste à dévoiler le phénomène de multi-engagement en étudiant les liens de co-affiliation susceptibles d'exister entre les huit associations de la cause des étrangers dans le Nord de la France étudiées. Par co-affiliation, nous entendons les liens qui unissent deux associations de par le fait qu'elles possèdent des militants en commun. Ainsi, dans le graphe de la Figure $1^{11}$, plus le lien qui relie deux associations est épais, plus les militants qui co-appartiennent à ces

\footnotetext{
${ }^{10}$ Dès le milieu des années 1940, la préoccupation pour le logement des travailleurs immigrés est centrale dans les actions sociales menées, notamment par des entreprises ou organisations patronales (notamment dans le domaine du textile ou des mines) mais aussi par des associations.

${ }^{11}$ Les graphes présentés dans cet article sont réalisés avec le logiciel libre Pajek. La matrice de base est de type 2-mode : en ligne les individus, et en colonne les organisations militantes. Elle est ensuite transformée en matrice carrée de type 1-mode : les organisations à la fois en ligne et en colonne.
} 
deux associations sont nombreux ; et à l'inverse, plus il est fin, moins sont nombreux les militants coaffiliés à celles-ci ; de même, certains liens n'existent pas, ce qui indique une absence totale de militants en commun. Les chiffres accompagnant chaque lien indiquent ici le nombre de militants en commun, le maximum étant de 7 entre le GRIAM et SALAM - deux associations d'action sociale ; on ne note, à l'inverse, aucun lien entre le CSP59 et le GRIAM ou la CIMADE. La taille des points est quant à elle proportionnelle au nombre de militants ayant répondu à notre questionnaire dans chaque association : il apparaît par exemple que la CLÉ et le CSP59, qui disposent respectivement de 106 et 73 militants, n'en ont qu'un seul en commun. D'une manière générale, cette analyse fait donc apparaître une très faible interdépendance des associations : peu de militants appartiennent simultanément à deux d'entre elles. En effet, si tous les militants interrogés $(n=389)$ appartenaient à toutes les associations représentées $(n=8)$, le nombre maximal de liens de co-affiliation possibles atteindrait 3112, or nous n'en observons que 33 . La densité du réseau est donc très faible $(1 \%)^{12}$.

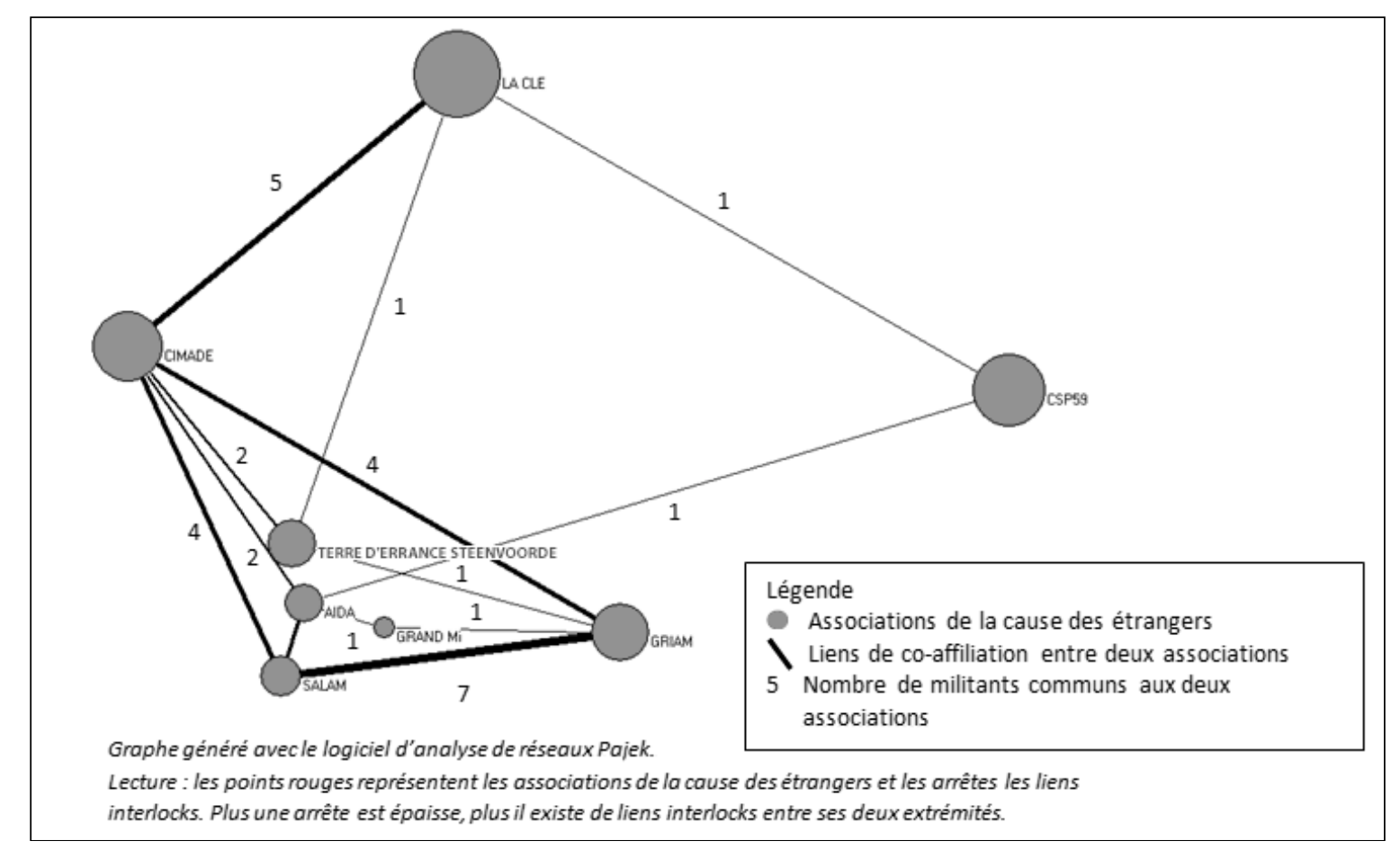

Figure 1 : Réseau de co-affiliation inter-associative de la cause des étrangers 1 -mode ${ }^{13}$

La Figure 2 est une autre façon d'appréhender ce phénomène de multi-engagement; les 389 militants y sont représentés ainsi que la ou les associations dont ils sont membres. L'intérêt de cette représentation est de faire explicitement apparaître les individus «passeurs », c'est-à-dire ceux qui sont simultanément co-affiliés à deux associations ou plus. Dans le cas présent, seuls vingt-cinq militants $(6,5 \%)$ sont dans ce cas.

\footnotetext{
${ }^{12}$ La densité se calcule en divisant le nombre de liens observés par l'enquête par le nombre de liens possibles si tous les points étaient reliés entre eux. Elle s'exprime en \%. Un \% élevé indique que beaucoup de liens sont observés dans le réseau, et inversement.

${ }^{13}$ Nos remerciements vont à Julien Lapasset pour son aide précieuse dans la mise en forme graphique des figures.
} 


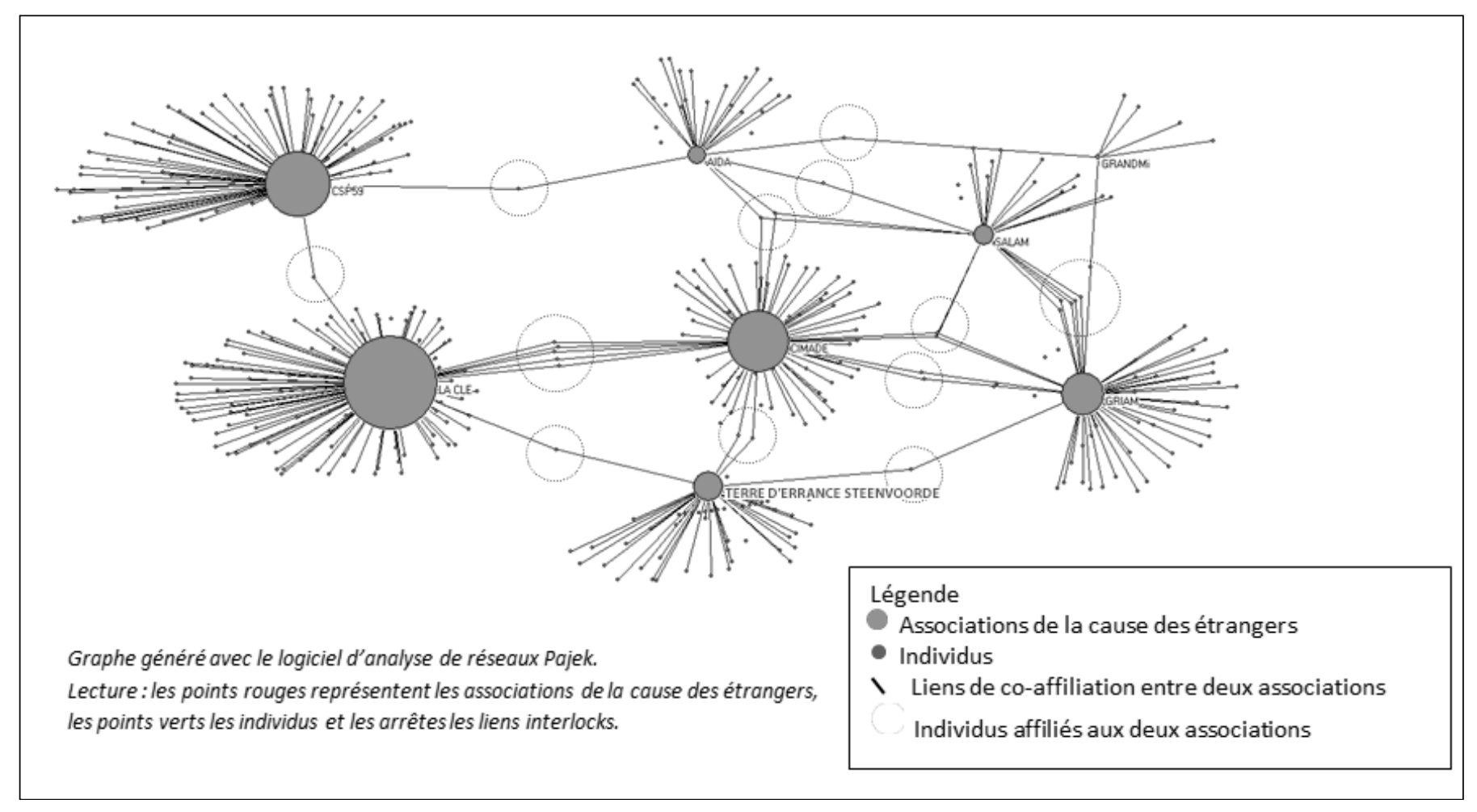

Figure 2 : Réseau de co-affiliation inter-associative de la cause des étrangers 2-mode

La faiblesse des liens inter-associations que mettent en lumière ces deux graphes s'explique selon nous par le processus historique de division du travail militant qui a abouti, comme nous l'avons vu plus haut, à la situation actuelle caractérisée par une forte spécialisation des associations sur l'une des quatre formes d'action que nous avons précédemment décrites. Cette première analyse, au sein de laquelle apparaissent peu de liens de co-affiliation, nous inviterait plutôt à conclure à une faiblesse de la pratique du multi-engagement et à un cloisonnement organisationnel de l'espace militant appuyé sur quelques formes d'action spécialisées. Cependant, à travers nos entretiens et nos observations de terrain, ainsi qu'à travers notre expérience militante, nous avions pu faire le constat que les pratiques de multi-engagement étaient répandues, et que de nombreux militants ne se dédiaient pas qu'à une seule cause. C'est la raison pour laquelle nous nous sommes efforcé de recueillir, de façon systématique au moyen d'un questionnaire, toutes les autres appartenances, syndicales, partisanes ou associatives (Encadré 2) des militants de la cause des étrangers, et les avons représentées au sein d'un même graphe, celui de la Figure 3. 


\section{Encadré 2 : Liste des organisations militantes les plus citées par les répondants au questionnaire ${ }^{14}$}

1. Les associations de la cause des étrangers

CLÉ, CSP59, CIMADE, GRIAM, Terre d'Errance Steenvoorde, SALAM, AIDA et Le Grand Mi.

\section{Les syndicats}

CFDT, CFTC, CGT, SUD, FSU, CNT.

\section{Les partis politiques}

PS, PCF, Europe Écologie-Les Verts, NPA, MODEM, Coordination communiste.

\section{Les autres associations}

MRAP, LDH, ATD, ATTAC, Secours populaire, Amnesty International, RESF, CCFD, Emmaüs, Secours catholique, Banque alimentaire, Pastorale des migrants, Terre solidaire, CCL, GDALE, No Border, APU, IDM, Collectif Afrique, France Cuba, AFPS, SOS Racisme, L’̂̂le aux livres, Lire et faire lire.

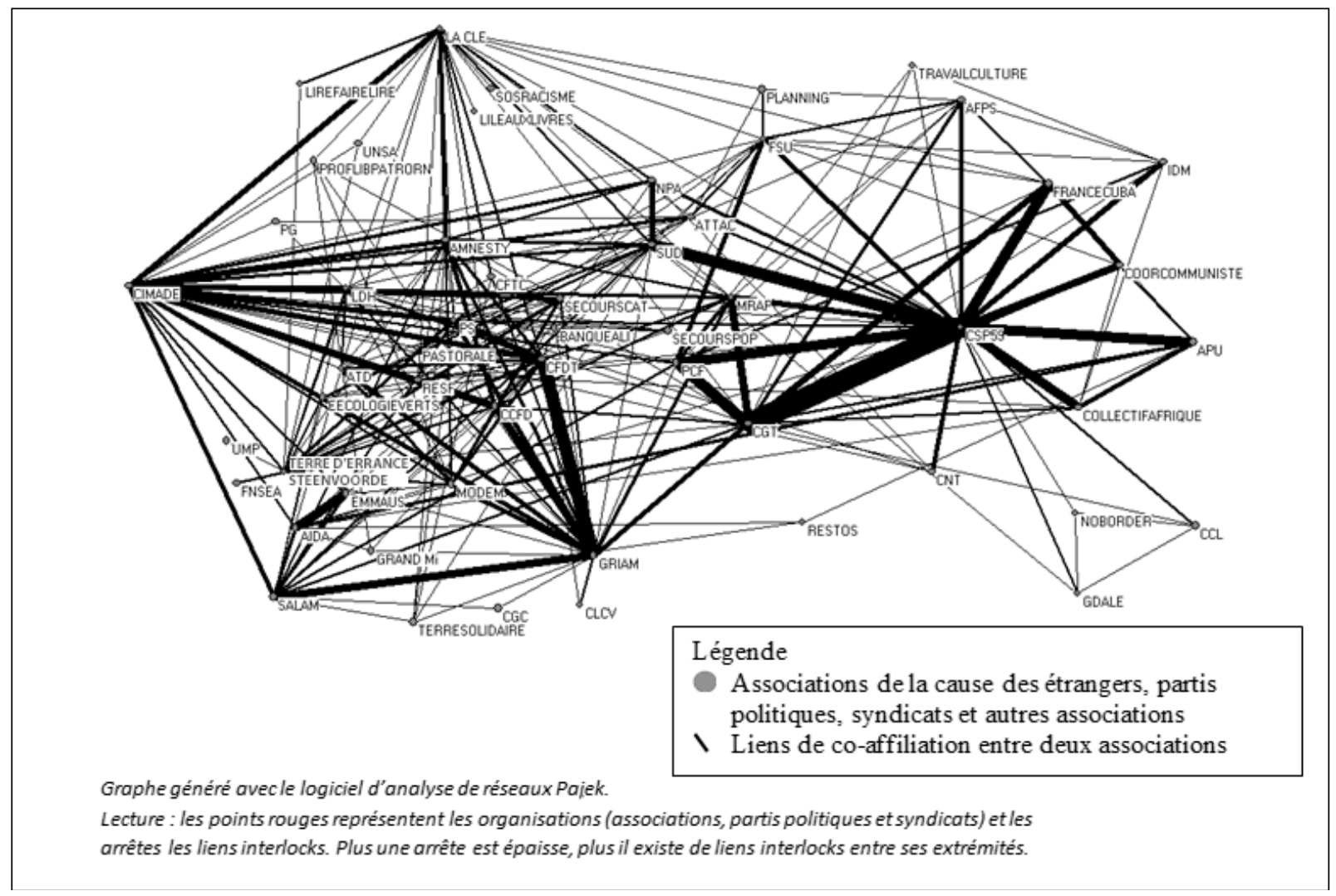

Figure 3 : Réseau de co-affiliation entre les associations de la cause des étrangers, les syndicats, les partis politiques et les autres associations

Par rapport aux figures 1 et 2, la figure 3 est beaucoup plus complexe (on passe de 8 à 44 organisations militantes) et dense $(17 \%$ contre $1 \%$ précédemment), faisant cette fois apparaître les pratiques de multi-engagement comme structurantes dans l'espace de la cause étudiée. Derrière une

${ }^{14}$ Voir la liste des acronymes en annexe. 
multiplicité de liens en apparence désordonnés, le graphe révèle un espace militant dont la structure organisationnelle n'est pas uniforme mais tend à s'articuler autour de deux pôles distincts. D'un côté (à la gauche du graphe), les liens de co-affiliation constitués autour de sept des huit principales associations de la cause des étrangers (AIDA, SALAM, Terre d'Errance Steenvoorde, Le Grand-Mi, le GRIAM, la CLÉ, la CIMADE), et de l'autre (à la droite du graphe), les liens de co-affiliation formés autour du CSP59. L'analyse des réseaux permet de faire apparaître encore plus clairement cette coexistence de deux pôles d'organisations en prenant le parti de ne retenir que les liens de co-affiliation représentant au minimum quatre individus (Figure 4). Nous remarquons alors qu'il n'existe que peu de liens, donc peu de militants qui font le pont entre les deux pôles d'organisations.

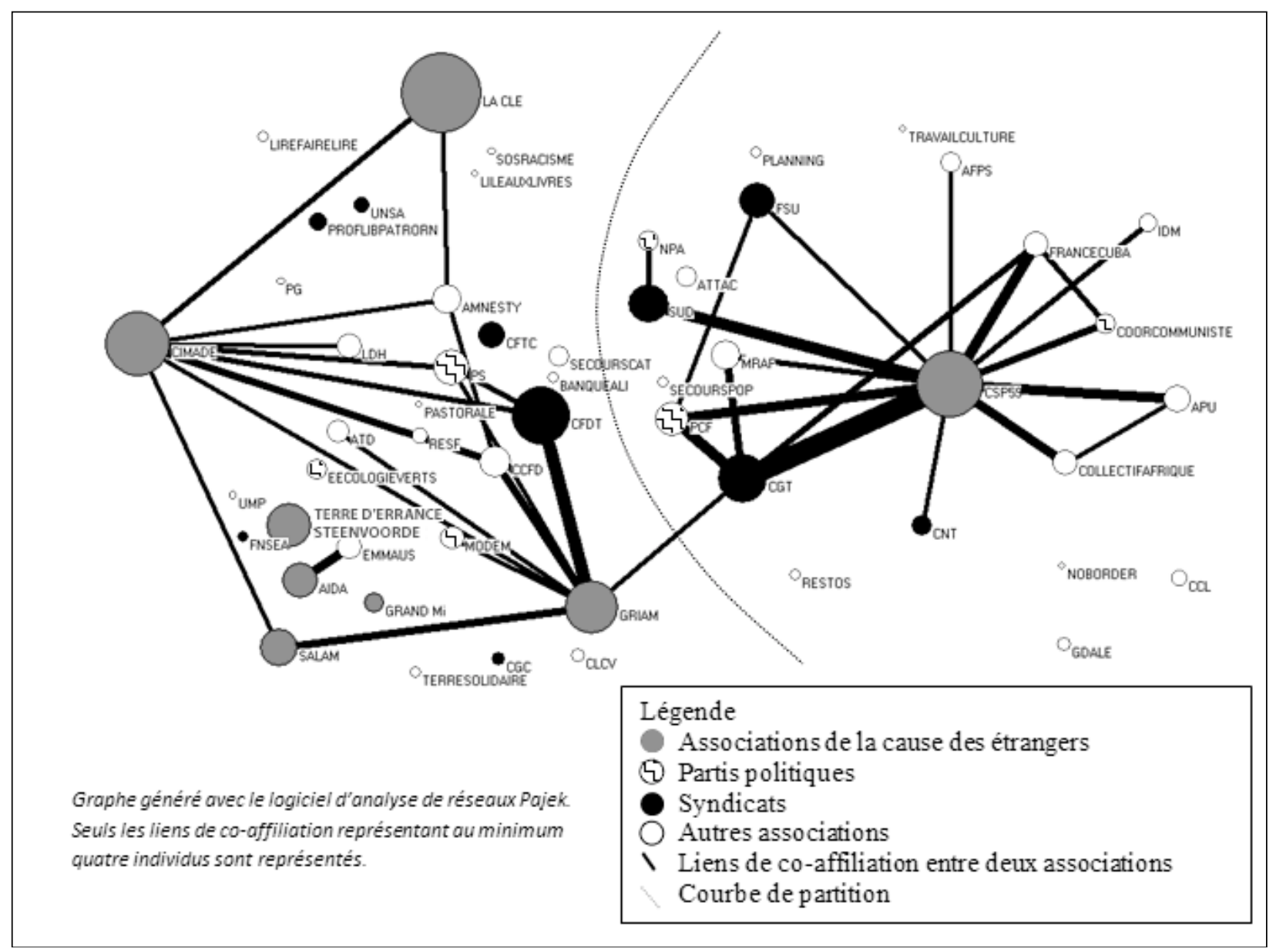

Figure 4 : Réseau de co-affiliation entre les associations de la cause des étrangers, les syndicats, les partis politiques et les associations

On peut alors tracer manuellement une courbe matérialisant cette bipolarité de l'espace militant : seul un lien ${ }^{15}$ traverse cette courbe, reliant la CGT au GRIAM ; en ce qui concerne les autres liens de coaffiliation, ils ne se concrétisent qu'entre organisations d'un même pôle. Ainsi, l'homogénéité du monde militant ne doit pas masquer une hétérogénéité interne. En effet, les deux pôles d'organisations que l'analyse de réseaux met en lumière au sein de la cause des étrangers, renvoient à des histoires, des idéologies et des conceptions distinctes de la figure de l'étranger. D'un côté (à la gauche du graphe), nous identifions un pôle chrétien au sein duquel est visible un sous-pôle du catholicisme social; et de l'autre (à

${ }^{15}$ Signifiant l'existence de quatre militants communs. 
la droite du graphe), nous reconnaissons un pôle d'extrême-gauche à dominante communiste. Même s'il existe entre eux des modes d'action « passerelle » (Encadré 3) et des militants «passeurs » (Encadré 4), ces deux pôles d'organisations sont relativement étanches. Pour chacun d'eux, l'analyse de réseaux révèle le rôle central et structurant d'une organisation syndicale, respectivement la CFDT et la CGT, qui permettent d'interroger, plus largement, la relation entre syndicalisme et immigration.

\section{Un pôle d'organisations chrétiennes...}

Le premier pôle identifié regroupe sept associations de la cause des étrangers sur les huit : il s'agit d'associations d'action sociale, d'alphabétisation et de défense des droits intervenant auprès des étrangers par le biais d'un suivi social, de distributions de repas, de vêtements ou de soins, de cours de français ou encore de conseil et d'accompagnement juridique. Ces associations ont en commun d'établir des liens de co-affiliation avec un ensemble d'organisations militantes proches des milieux chrétiens. C'est le cas d'organisations syndicales (la CFDT et la CFTC), de partis politiques (le PS et dans une moindre mesure Europe Ecologie Les Verts) et d'associations (le Secours Catholique, Amnesty International, ATD Quart Monde, CCDF Terre solidaire ou encore Emmaüs). Parmi elles, certaines relèvent davantage d'un souspôle du catholicisme social, dont les militants - plus couramment qualifiés de " cathos de gauche » - sont membres d'organisations telles que la CFDT, du CCFD Terre solidaire ou encore d'ATD Quart Monde. Si la proximité de la CIMADE avec les organisations catholiques peut paraître étonnante au regard de l'histoire protestante de l'organisation, elle est en réalité due à la composition actuelle des militants de l'organisation, qu'ils soient bénévoles ou salariés. Parmi les militants de la CIMADE ayant répondu à l'enquête, $39 \%$ se déclarent catholiques ce qui représente trois militants sur quatre parmi ceux s'étant déclarés croyants.

Cette influence des organisations chrétiennes se manifeste par exemple par le soutien de personnalités lors de mobilisations sociales telles que des grèves de la faim d'étrangers sans-papiers. On pense notamment à l'Abbé Pierre, à Jacques Gaillot (créateur de l'association Droit Devant !!) ou plus récemment à Alain Richard, franciscain et initiateur à Toulouse en 2007 des Cercles du silence visant à dénoncer les conditions de rétention des étrangers dans les Centres de rétention administrative. Historiquement, la tradition militante chrétienne a donné lieu à la création d'associations spécifiques faisant partie du " mouvement de solidarité d'inspiration chrétienne » (Mathieu, 2009, p.39). Les années 1960 sont en effet marquées par l'engagement des « cathos de gauche » dans les mobilisations politiques (Rousseau 2008 ; Pelletier, Schlegel, 2012), que ce soit dans le militantisme partisan (création du PSU en 1960), syndical (création de la CFDT en 1964) ou lors de mobilisations collectives (en faveur de l'indépendance de l'Algérie ou lors des grèves de 1968 par exemple). Cet engagement se poursuit dans les années 1970 : des chrétiens participent à la mobilisation des Lip, au mouvement pour la défense du Larzac ainsi qu'à des grèves de la faim de sans-papiers (Siméant, 1998a). L'engagement auprès des étrangers des militants chrétiens, et plus précisément de ceux marqués par le catholicisme social, s'inscrit dans cette dynamique et, plus largement, dans l'héritage de la philanthropie (Havard Duclos, 2002 ; Havard Duclos, Nicourd, 2005).

Dans ce cadre, l'étranger est perçu comme l'incarnation de l'altérité, autrement dit le plus autre des autres. La figure de l'altérité est principalement partagée par les militants et organisations proches du vivier du militantisme chrétien. L'étranger est dans ce cadre considéré comme l'incarnation même de l'altérité, autrement dit le plus autre des autres. S'il faut l'aider et l'accueillir, c'est parce que tous les individus font partie d'une même famille, celle de l'Humain. Altérité et humanité constituent ainsi un binôme structurant ce que Johanna Siméant nomme "la sensibilité chrétienne » (Siméant, 1998a, p.182). Pour les militants qui se rattachent à cette figure de l'étranger, c'est le caractère humain qui est mis en avant et évoqué régulièrement lors des entretiens. Plus précisément, c'est parce que l'étranger - cet autre 
faisant partie de la même humanité - souffre, qu'il faut l'aider et l'accueillir. C'est donc dans une vision empathique et en tant qu'individu que l'étranger est perçu ici.

$\mathrm{Au}$ sein de ce premier pôle d'organisations, la CFDT apparaît centrale : parmi les militants syndiqués au moment de l'enquête ( $28 \%$ des enquêtés), près d'un sur quatre est membre de la CFDT. Il s'agit du syndicat co-affilié au plus grand nombre d'associations de la cause des étrangers puisque toutes, à l'exception du CSP59, ont dans leurs rangs des militants membres de la CFDT. Cette centralité peut paraître étonnante au regard de l'enquête de terrain, dans la mesure où la CFDT n'apporte aucun soutien, officiel ou officieux, aux mobilisations sociales en faveur des étrangers. En réalité, cette centralité s'explique exclusivement par les phénomènes de multi-engagement: comme le montre l'analyse de réseaux, les militants de la CIMADE et du GRIAM sont très nombreux à être syndiqués à la CDFT. Les pratiques d'engagements multiples s'inscrivent dans un processus spécifique d'encastrement organisationnel : du fait de leur socialisation, les militants sont ancrés dans le pôle chrétien, et plus précisément dans le catholicisme social, et sont de ce fait davantage conduits vers une syndicalisation à la CFDT plutôt que vers tout autre syndicat. La position de la CFDT dans le graphe correspond donc moins à un engagement actuel du syndicat qu'à un engagement historique, issu de cette tradition chrétienne et catholique sociale du militantisme (Andolfatto, Labbé, 2000, p.34). C'est par exemple le cas entre 1972 et 1975 lors des grèves de Tunisiens et Marocains, quand certains militants du Mouvement des travailleurs arabes se tournent vers la CFDT (Siméant, 1998a, p. 96) ainsi qu'en 1980, dans le quartier du Sentier, à l'occasion de la grève de la faim menée par des travailleurs turcs employés dans la confection (Siméant, 1998a, p. 16 ; Galano, Spire, 2002 ; Mathieu, 2009, p. 39); 1'Union départementale de Paris s'engage alors activement dans le soutien aux grévistes et de nombreux sans-papiers se syndiquent à la CFDT. Selon Siméant, le soutien de la CFDT, loin de relever d'une prise de position de la centrale syndicale, se manifeste soit par le biais de militants syndiqués engagés dans des mobilisations locales, soit par le biais d'Unions locales engagées ponctuellement, soit enfin par le biais de branches plus radicales ${ }^{16}$ du syndicat qui tentent d'engager la centrale dans un soutien national. Le soutien de la CFDT en faveur des étrangers sans-papiers n'est donc pas un engagement confédéral mais relève bien plus de fractions du syndicat qui se situent le plus souvent dans une relation singulière à la confédération et à la direction nationale (Siméant, 1998a, p. 167). Ces fractions sont souvent proches de la tradition autogestionnaire du syndicat, héritée de plusieurs luttes des années 1970, telle celle des Lip (Mathieu, 2009, p.29). Cependant aujourd'hui, et malgré cet engagement historique de la CFDT, ce syndicat apparaît totalement absent des luttes revendicatives en faveur des étrangers dans le Nord de la France.

Au-delà des pratiques d'engagements multiples dans des organisations chrétiennes, l'influence religieuse se manifeste aussi par son rôle dans la socialisation des militants. Plus de $50 \%$ des militants interrogés déclarent avoir une religion et parmi eux, plus de $85 \%$ sont catholiques, six sur dix se disent pratiquants et $30 \%$ ont des responsabilités religieuses (prêtre, religieuse, animation en pastorale, catéchisme, etc.). Les engagements antérieurs au moment de l'enquête dans des organisations dites de jeunesse confirme ce rôle socialisateur : parmi ceux ayant été engagés dans un mouvement de jeunesse (32\% des personnes interrogées), 83\% l'ont été en effet dans une organisation d'obédience catholique.

\footnotetext{
${ }^{16}$ L'auteur précise qu'il s'agit notamment de branches de la CFDT qui participeront plus tard activement à la création des syndicats SUD.
} 


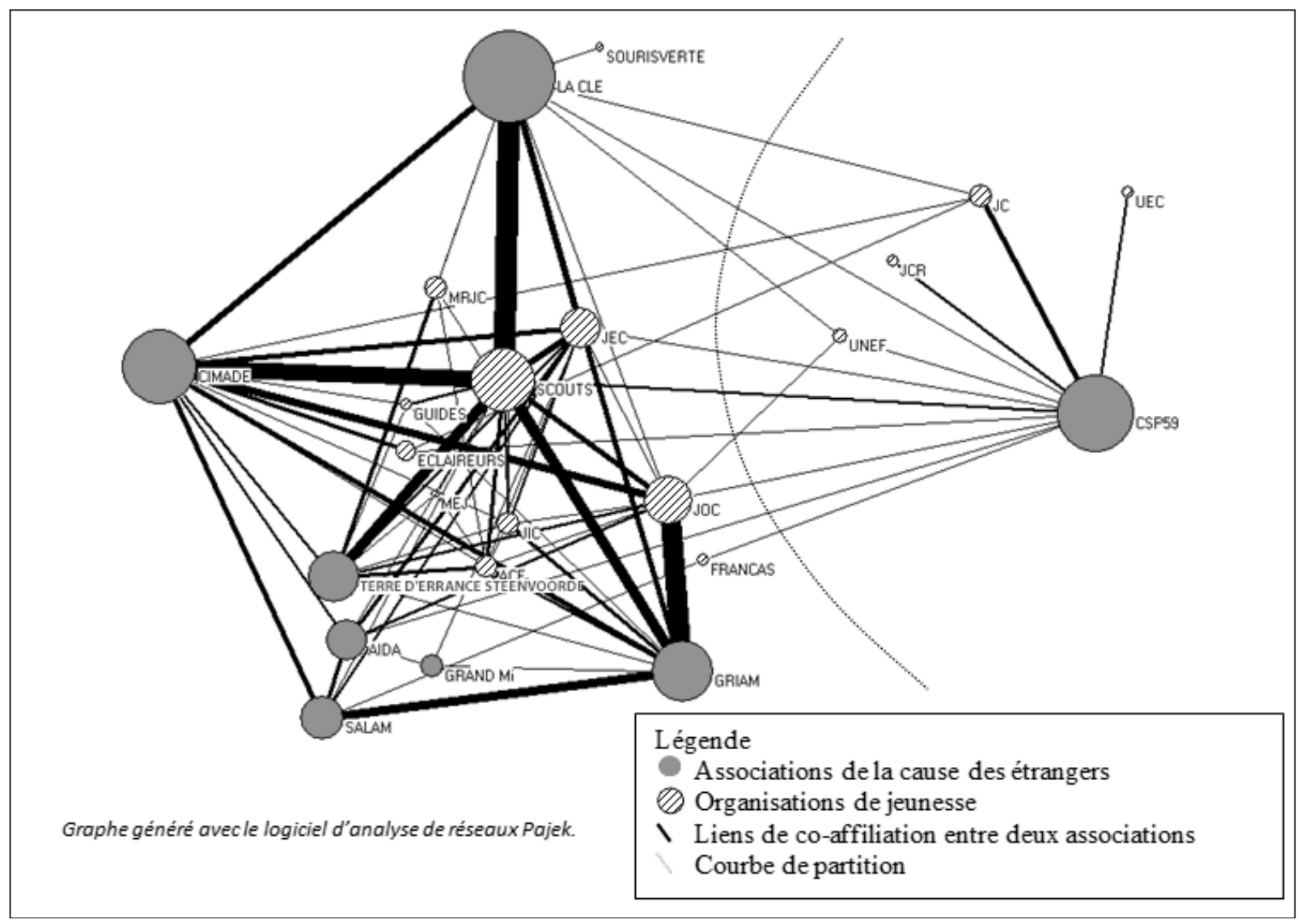

Figure 5 : Réseau de co-affiliation entre les associations de la cause des étrangers et les organisations de jeunesse

Le réseau de co-affiliations entre les associations de la cause des étrangers et les mouvements de jeunesse (Figure 5) confirme la centralité des organisations chrétiennes et le rôle de la socialisation religieuse dans les parcours d'engagement des militants inscrits dans ce premier pôle du réseau. En effet, les trois organisations de jeunesse les plus représentées, les Scouts de France, la JOC et la JEC, sont d'obédience catholique. À l'intérieur du pôle chrétien, le graphe permet de repérer des organisations catholiques (les Scouts et les Guides de France, l'ACE ou la MEJ qui représentent $46 \%$ des engagements déclarés dans des mouvements de jeunesse) ainsi que des organisations d'action catholique (la JOC, la JEC, les JIC pour $37 \%$ d'entre eux) faisant partie du sous-pôle du catholicisme social. Si les Scouts sont centraux dans ce réseau, dans la mesure où ils sont co-affiliés à toutes les associations de la cause des étrangers, leur lien privilégié avec la CIMADE et la CLÉ confirme la proximité de ces deux associations avec le pôle chrétien. En entretenant des liens plus forts avec la JOC, le GRIAM s'ancre quant à lui dans le sous-pôle du catholicisme social. 


\section{Encadré 3 : Deux modes d'action « passerelles »}

Interrogés par l'intermédiaire du questionnaire sur leurs pratiques militantes ${ }^{17}$, les vingt-cinq militants repérés comme "passeurs » (affiliés à plusieurs associations de la cause, Figure 2) déclarent mener des activités juridiques auprès des étrangers. Il s'agit en effet du seul mode d'action qui est commun à toutes les associations de cette cause. Lors des permanences juridiques organisées par les salariés et bénévoles de chaque associations, les mêmes outils, connaissances et compétences, sont mobilisés. L'usage du droit au service de la cause apparaît ainsi comme une pratique qui uniformise et routinise le travail militant (Pette, 2014). Mais c'est aussi un mode d'action qui facilite les échanges entre les militants de différentes associations : les militants les plus investis dans l'aide juridique se téléphonent de temps en temps au sujet de dossiers qu'ils suivent ensemble ou de cas de jurisprudences, et les associations organisent parfois des formations juridiques ensemble qui sont l'occasion pour leurs militants de se rencontrer et d'échanger sur leurs pratiques.

De plus, parmi ces militants, nombreux sont ceux qui ont participé en tant que militants « soutiens ${ }^{18}$ à des grèves de la faim d'étrangers sans-papiers. Si ce mode d'action trouve son origine dans l'expérience de Gandhi et des mouvements non-violents (Siméant, 2009), son usage à partir des années 1960 dans les mobilisations collectives en fait l'héritier à la fois du militantisme chrétien de gauche et du militantisme d'extrême-gauche. Organisées dans le Nord de la France par des militants proches des organisations communistes, les grèves de la faim reçoivent aussi le soutien actif des militants chrétiens qui se disent sensibles à la mise en danger, par les sans-papiers, de leur propre vie. La souffrance du corps (dans son acceptation physique) qui ne s'alimente plus devient, dans ces circonstances, le symbole de la souffrance du corps (dans son acceptation sociale) des sans-papiers qui n'ont pas d'existence légale.

L'action juridique et les grèves de la faim apparaissent ainsi à l'observateur comme deux modes d'action "passerelle », propices à la rencontre entre des militants d'organisations et de traditions militantes distinctes ainsi qu'à leur circulation dans l'espace de la cause, même si celle-ci reste faible.

\section{... face à un pôle d'organisations d'extrême-gauche à dominante communiste}

Le second pôle d'organisations est structuré différemment puisqu'il n'est centré qu'autour d'une seule association, le CSP59, qui mobilise un répertoire d'action caractéristique des luttes revendicatives : manifestations, marches, occupations de lieux symboliques ou encore grèves de la faim par exemple. Cette organisation semble à ce titre occuper une place à part : il s'agit à la fois de l'association la moins coaffiliée aux autres associations de la cause des étrangers (Figure 1), et celle la plus co-affiliée à des syndicats, partis politiques et associations (de défense des causes et des droits, notamment), qui n'entretiennent eux-mêmes que peu de liens avec les sept autres associations du réseau. Cette singularité est évoquée dans plusieurs entretiens par les militants sous les expressions " galaxie CSP », " le monde du CSP59 » ou « petit monde ». Cette place à part dans le réseau ne signifie pas pour autant qu'il n'y est pas central : il bénéficie au contraire d'une position de monopole en ce qui concerne la forme d'action dite des luttes revendicatives, et ses nombreux militants «multicartes » lui assurent une place structurante dans l'espace de la cause des étrangers (Duriez, 2004).

Ce second pôle relie entre elles des organisations principalement communistes : le CSP59 est en effet co-affilié au PCF et à la Coordination communiste (revendiquant la reconstruction d'un parti marxiste-léniniste et faisant partie du Rassemblement des cercles communistes), à des associations telles

\footnotetext{
${ }^{17}$ Il s'agit d'une question ouverte.

${ }^{18}$ Expression indigène.
} 
que le MRAP, le Secours Populaire et des associations de soutiens politiques à Cuba et à la Palestine. Dans cette configuration locale, la tradition militante d'extrême-gauche se traduit par un pôle communiste prédominant dans les mobilisations collectives relatives à la cause des étrangers ${ }^{19}$, qui tend à invisibiliser d'autres fractions traditionnelles de l'extrême-gauche, telles que les organisations trotskystes, antifascistes, alternatives ou anarchistes et autonomes (Sommier, 2002). Ces dernières sont néanmoins visibles sur le graphe avec des groupes tels que le Groupes des anarchistes de Lille et environs, ou le collectif No Border particulièrement actif dans le Nord de la France suite à l'organisation d'un camp à Calais en juin 2009.

L'ensemble des associations représentées dans ce second pôle sont particulièrement visibles au sein de l'espace militant étudié lors de mobilisations sociales ; par l'investissement ponctuel de militants, par la signature de tracts ou par la mise à disposition de ressources matérielles (des locaux pour la tenue d'une réunion, la reproduction de tracts, la participation financière lors d'organisation d'événements ou la tenue de caisses de grèves par exemple). Contrairement au pôle chrétien, les organisations de jeunesse sont ici peu centrales (Figure 5) : celles citées sont des organisations syndicales et politiques (1'UEC, des JC, des JCR et de l'UNEF) et ne représentent que $9 \%$ des engagements déclarés dans des organisations de jeunesse $^{20}$.

Les étrangers sont ici davantage perçus comme l'une des populations les plus dominées, qui doit par conséquent recevoir toute leur attention. Parmi les étrangers, ce sont les sans-papiers qui représentent l'archétype des populations dominées. Ils sont en effet considérés comme les plus dominés et les plus exploités, les plus prolétaires des prolétaires. Johanna Siméant souligne que les militants d'extrêmegauche estiment trouver ici « la quintessence du prolétariat» (Siméant, 1998a, p.182). Cette figure de l'étranger s'inscrit dans une lecture critique du capitalisme qui en dénonce les conséquences négatives. Alors que les militants chrétiens se disent sensibles à la souffrance des immigrés, celles et ceux qui s'inscrivent dans le militantisme d'extrême-gauche mettent au centre de leurs discours la notion d'égalité des droits.

En ce qui concerne la pratique syndicale des militants, ce second pôle d'organisations est quant à lui co-affilié à quatre syndicats : la CGT (28\% des militants syndiqués au moment de l'enquête), SUD $(16,5 \%)$, et dans une moindre mesure, la FSU (9\%) et la CNT. Ces quatre organisations syndicales sont systématiquement signataires des appels à la mobilisation; elles sont visibles dans les cortèges par les badges ou drapeaux portés par les manifestants. Par ailleurs, les militants syndicalistes mettent régulièrement des ressources à disposition du CSP59 (impressions de tracts, locaux pour des réunions ou cotisations lors d'événement tels que des concerts) et cinq grèves de la faim se sont déroulées à Lille sur le site de la Bourse du travail entre 1999 et 2007. Parmi ces quatre organisations syndicales, c'est la CGT qui apparaît cette fois-ci la plus centrale : elle entretient avec le CSP59 le lien de co-affiliation le plus fort, et est le syndicat auquel sont adhérents les leaders du CSP59. La CGT, second syndicat le plus représenté, entretient vis-à-vis de l'immigration un rapport historique ambigu et variable. En effet, pendant les Trente Glorieuses, la CGT voit dans l'immigration une armée de réserve industrielle du patronat. En fournissant un réservoir de main-d'œuvre prête à accepter des conditions de travail précaires et une rémunération au rabais, l'immigration mettrait en danger le rapport de force entre travail et capital. La main-d'œuvre immigrée est donc perçue par certains militants comme une concurrence déloyale à la main d'œuvre nationale (Siméant, 1998a, p. 165), la fermeture des frontières intervenant dans ce contexte comme une solution face à la fragilisation des conditions de travail (Barron et alii, 2011, p. 292). Cette position d'hostilité vis-à-vis des travailleurs étrangers est néanmoins couplée avec un principe de solidarité internationale de classe. Issue du mouvement ouvrier (Andolfatto, Labbé, 2000, p. 22) et située au sein d'une « constellation idéologico-politico-associative centrée sur le PCF » (Contamin, Delacroix, 2009, p.82), la CGT s'est ainsi trouvée prise dans des tensions entre la figure de l'ouvrier et celle de l'immigré

\footnotetext{
${ }^{19}$ Au sein du champ du militantisme de gauche et d'extrême-gauche défini par Cécile Péchu (2006), ces organisations relèvent des pôles organisationnel et classiste (respectivement opposés aux pôles spontanéiste et contre-culturel). Les frontières de l'extrême-gauche sont susceptibles d'être modifiées selon les contextes locaux.

${ }^{20}$ Ce chiffre ne représente que les engagements déclarés dans des organisations dites de jeunesse et ne permet pas de mesurer tous les expériences militantes de jeunesse des militants interrogés. On peut faire l'hypothèse qu'au sein du pôle d'organisations communistes, la socialisation militante s'est faite par le biais d'organisations militantes plus classiques telles que le PCF.
} 
(Pitti, 2001). Au cœur de ces débats, les logiques d'intégration et d'exclusion entrent alors en contradiction (Dedieu, 2011, p.146). De plus, le soutien de la CGT aux étrangers sans-papiers n'est pas le fait de tous les militants cégétistes. Si les grèves de travailleurs sans-papiers ont été largement soutenues et organisées par la CGT en 2008 et 2009 dans la région parisienne (Barron et alii, 2011), les prises de position des dirigeants du syndicat ainsi que des militants ne vont pas toutes dans le sens de la défense des travailleurs sans-papiers. Des conflits persistent entre les partisans de la régularisation de tous les sans-papiers, ceux préférant revendiquer la régularisation des travailleurs sans-papiers, et ceux adeptes de positions plus restrictives en matière d'immigration.

\section{Encadré 4 : Colette, une trajectoire à la jonction des deux pôles d'organisations}

Les deux pôles d'organisations présentés ici fonctionnent aussi comme viviers de recrutement des militants. Si les récits de vie réalisés révèlent que les trajectoires militantes se déroulent le plus souvent dans un seul pôle d'organisations, certains cas plus atypiques, comme celui de Colette, 66 ans, retraitée, ancienne professeure agrégée d'arts plastiques dans le secondaire, se situent à la jonction des deux viviers de recrutement.

Quand je la rencontre en 2009, Colette organise des permanences juridiques au MRAP et participe régulièrement aux manifestations du CSP59. Elle est née près de Béthune de parents catholiques, enseignants et engagés dans les groupes de Témoignage chrétien et dans les réseaux de soutien aux indépendantistes algériens. Alors que son grand-père est syndiqué à la CGT, son père rejoint le SGEN. Elle s'engage pour la première fois à la JEC avant de rejoindre la JOC. "Après, la suite, c'est la fac et Mai 68. J'ai vécu 68 avec des gens qui étaient plus ou moins proches de l'UNEF. À l'UNEF, ça commençait déjà à ruer dans les brancards. Parmi eux, certains se sont rapprochés peu à peu des groupes d'extrême gauche, du PCML. J'ai travaillé avec eux dans un atelier d'arts plastiques et à partir de là, je suis rentrée au PCML. J'ai connu l'époque des anciens du Secours rouge. Tout ce noyau-là... Sur la fac, j'étais engagée au niveau du syndicat [au SGEN] - c'était l'époque de l'autogestion, pas comme maintenant! et après la fin de mes études, je suis restée en contact avec des militants de l'université et on militait au comité de défense des étudiants étrangers. " Après avoir participé à un collectif antiraciste, elle rejoint le MRAP en 1978 et suit des formations au droit des étrangers à Paris avec le GISTI. À la même époque, elle quitte le SGEN et se syndique à la CGT où elle est toujours aujourd'hui. Colette vote pour le Front de gauche, est adhérente au MRAP et à l'Association France Palestine Solidarité et se rend de temps en temps aux Cercles $d u$ Silence organisés par des organisations catholiques.

Les proximités de Colette avec les deux pôles d'organisations ont ainsi évolué au cours de son parcours personnel et professionnel. Ce récit révèle non seulement le rôle des événements personnels et historiques dans les bifurcations des trajectoires militantes, ici symbolisés par l'entrée à l'université et l'expérience des mobilisations de Mai 68, mais aussi les phénomènes de continuité qui peuvent exister entre le militantisme chrétien et le militantisme d'extrême-gauche.

\section{Vers une représentation graphique de l'espace militant}

En mêlant approches quantitative et qualitative pour étudier les engagements multiples des militants engagés dans la cause des étrangers, on aboutit à une représentation graphique de l'espace militant étudié. La Figure 6 représente le réseau d'affiliations entre les associations de la cause des étrangers et l'ensemble des autres catégories d'organisations auxquelles sont affiliés, au moment de l'enquête, les militants que nous avons interrogés. Mais nous avons aussi ajouté, sur le même graphe, les organisations de jeunesse dont les militants ont fait partie auparavant. Les pôles d'engagement y apparaissent clairement : à gauche du graphe, le pôle chrétien (à l'intérieur duquel on peut repérer plus précisément le pôle du catholicisme social) et à droite, le pôle communiste prédominant dans la 
configuration locale de l'extrême-gauche. Dans une moindre mesure, les organisations anarchistes et autonomes sont aussi repérables sur le graphe. Est également visible sur le graphe la césure entre les différents pôles d'engagement et le peu de liens de co-affiliations qui existent entre eux. Par le biais des pratiques d'engagement multiple des militants, les techniques d'analyse des réseaux permettent ainsi d'objectiver les liens entre les organisations et d'aboutir à une représentation graphique de l'espace militant.

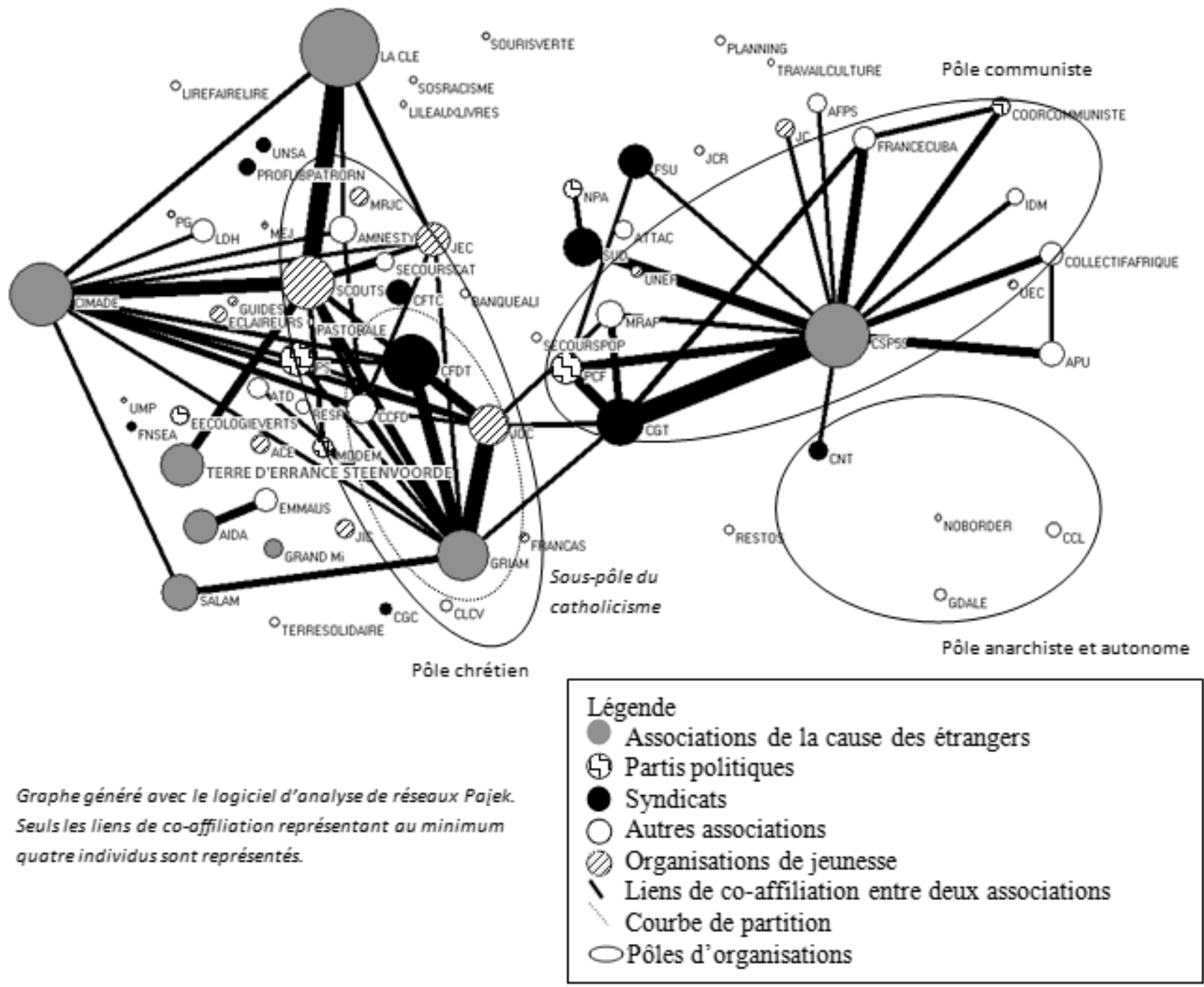

Figure 6 : Réseau de co-affiliation entre les associations de la cause des étrangers, les syndicats, les partis politiques, les associations et les organisations de jeunesse.

La cause des étrangers, tout comme d'autres mobilisations «des marges » (Crettiez, Sommier, 2002) ${ }^{21}$, se voit donc simultanément investie par des militants chrétiens et des militants d'extrême-gauche (Siméant, 1998a ; Lechien, 2003) ici saisis dans une configuration spécifique à la situation, à l'histoire locale. Chacun de ces deux pôles se caractérise par un ensemble d'organisations militantes formant un tout cohérent, comprenant un ou plusieurs syndicats, mouvements de jeunesse, partis politiques ${ }^{22}$ et autres

\footnotetext{
${ }^{21}$ Telles que les mobilisations pour le droit au logement (Péchu, 2006) ou celles des chômeurs (Mouchard, 2002).

22 À l'exception du pôle anarchiste et autonome.
} 
associations, ayant en commun de nombreux militants multi-engagés. Le pôle chrétien, dont fait partie le sous-pôle du catholicisme social, est ainsi composé par exemple des Scouts, de la JOC, de la CFDT, de la CFTC, du PS, du Secours catholique, d'ATD Quart monde ou du CCFD ; le pôle communiste est quant à lui composé entre autres de la CGT, du PCF ou du MRAP ; et le pôle anarchiste et autonome, de la CNT, du groupe local des anarchistes ou encore des groupes No Border. Cette cohérence interne des pôles résulte de processus d'encastrement organisationnel différenciés. Alors que le pôle chrétien s'appuie sur un invariant religieux qui se traduit par une permanence des organisations nationales au niveau local, les pôles communiste et anarchiste et autonome se déploient, quant à eux, davantage dans des configurations politiques qui varient selon les contextes locaux.

L'analyse des réseaux sociaux se révèle donc être un outil efficace pour appréhender la structure d'un espace militant. On voit notamment ici en quoi les liens de co-affiliation ne sont pas réductibles à la seule addition de choix individuels contingents, mais qu'ils constituent la manifestation de l'existence de processus d'encastrement organisationnel liés aux principaux pôles d'engagement, eux-mêmes préexistants à l'émergence d'une cause ou d'une lutte spécifique. En représentant les engagements multiples des militants, nous avons été en mesure de mettre au jour les pôles d'organisations et d'interroger de ce fait la façon dont s'articulent entre elles des constellations d'organisations.

Plus fondamentalement, la dimension structurale de cette recherche se donne à voir par la façon dont s'organise une division du travail au sein de l'espace de la cause des étrangers. Ainsi, alors que les associations plus proches du pôle chrétien s'engagent davantage dans l'action sociale, l'alphabétisation et la défense des droits, les associations plus proches du pôle communiste investissent quant à elles plus souvent les luttes revendicatives. L'enquête de terrain révèle que ces deux sous-espaces sont simultanément pris dans des relations de conflictualités et d'interdépendances. La ligne de fracture très nette que nous avons observée (Figures 3 à 6) ne doit pas masquer le fait que toutes les associations interviennent auprès d'une seule et même population - les étrangers - qui eux-mêmes circulent au sein de l'espace de la cause et créent de ce fait des liens entre les associations. Ces liens, qui sont révélés ethnographiquement lors de l'hommage au prêtre engagé Gérard Naissant, sont également éprouvés au moyen de l'analyse des réseaux sociaux du multi-engagement. C'est finalement une forme de solidarité organique qui, selon nous, semble se mettre en place, au fil du temps, entre les deux pôles d'organisation et leurs formes d'action. Complémentaires, les associations semblent, en effet, apporter ensemble une réponse globale à une même cause, qu'il s'agisse de demandes matérielles ou juridiques des étrangers ou d'enjeux politiques et législatifs, ceux de la politique migratoire.

Dans un souci de poursuivre le développement de cette perspective de recherche en sociologie de l'engagement et du militantisme, il serait possible de mobiliser la technique de l'analyse des réseaux pour réaliser une étude dynamique et historicisée de la configuration de l'espace militant. Pour cela, les données recueillies auprès des militants devraient rendre compte de manière exhaustive de leurs engagements présents et passés, de la succession de ces engagements et des périodes temporelles concernées. La structure de l'espace militant pourrait ainsi être analysée au regard, cette fois, des transferts d'une organisation à une autre, à l'intérieur même d'un pôle d'engagement ou entre deux pôles d'engagement. La représentation graphique de tels liens, orientés, et l'analyse séquentielle des données seraient en ce sens heuristiques pour comprendre dans quelle mesure la structure d'un espace militant est le résultat d'une construction historique. 


\section{Annexe Liste des acronymes des organisations présentes dans l'analyse des réseaux}

ACE Action catholique des enfants

AFSP Association France solidarité Palestine

AIDA Aide à l'insertion des demandeurs d'asile

APU Atelier populaire d'urbanisme

ATD Agir tous pour la dignité

ATTAC Association pour la taxation des transactions financières et pour l'action citoyenne

CCFD Comité catholique contre la faim et pour le développement

CCL Centre culturel libertaire

CFDT Confédération française démocratique du travail

CFTC Confédération française des travailleurs chrétiens

CIMADE Comité inter-mouvements auprès des évacués

CGT Confédération générale du travail

CLÉ Compter, lire, écrire

CNT Confédération nationale du travail

CSP59 Comité des sans-papiers du Nord

FSU Fédération syndicale unitaire

GDALE Groupe des anarchistes de Lille et environs

GRIAM Groupe de réflexion interreligieux d'aide aux migrants

IDM Immigration et droits des migrants

JC Jeunesses communistes

JCR Jeunesses communistes révolutionnaires

JEC Jeunesses étudiants chrétiennes

JIC Jeunesse indépendante chrétienne

JOC Jeunesse ouvrière chrétienne

LDH Ligue des droits de l'homme et du citoyen

MEJ Mouvement eucharistique des jeunes

MODEM Mouvement démocrate

MRAP Mouvement contre le racisme et pour l'amitié entre les peuples

NPA Nouveau parti anticapitaliste

PCF Parti communiste français

PCML Parti communiste marxiste léniniste

PS Parti socialiste

RESF Réseau éducation sans frontière

SALAM Soutenons, aidons, luttons, agissons pour les migrants et les pays en difficulté

SUD Solidaires, unitaires, démocratiques

UEC Union des étudiants démocratiques

UNEF Union nationale des étudiants de France

\section{Bibliographie}

AGRIKOLIANSKY É., 2002 La Ligue française des droits de l'homme et du citoyen depuis 1945. Sociologie d'un engagement civique. Paris : L'Harmattan.

ANDOLFATTO D., LABBÉ D., 2004 Sociologie des syndicats. Paris : La Découverte. 
BARRON P., BORY A., CHAUVIN S., JOUNIN N., TOURETTE L., 2011 On bosse ici, on reste ici ! La grève des sans-papiers : une aventure inédite. Paris ; La Découverte.

BERNARDOT M., 1999 Chronique d'une institution: la Sonacotra (1956-1976). Sociétés Contemporaines, $\mathrm{n}^{\circ} 33-34$, p. 39-58.

BERNARDOT M., 2008 Loger les immigrés. La Sonacotra 1956-2006. Paris : Éditions du Croquant.

BOUBEKER A., HAJJAT A., (dir.) 2008 Histoire politique des immigrations (post)coloniales. France, 1920-2008. Paris : Éditions Amsterdam.

BOURDIEU P., 1993 La misère du monde. Paris : Seuil.

BREIGER R. L., 1974 The Duality of Persons and Groups. Social Forces, vol.53, n² 2, p.181-190.

BRODIEZ A., 2006 Le Secours populaire français 1945-2000. Du communisme à l'humanitaire. Paris : Presses de Sciences Po.

CRETTIEZ X., SOMMIER I., (dir.). 2002 La France rebelle. Tous les foyers, mouvements et acteurs de la contestation. Paris : Michalon.

COMBES H., 2009 Pour une sociologie du multi-engagement: réflexion sur les relations partismouvements sociaux à partir du cas mexicain. Sociologie et sociétés, vol.41, n², p. 161-188.

CONTAMIN J-G., DELACROIX R., 2009 Les transformations des formes d'engagement au prisme du local. L'exemple de la CGT du Nord. Politix, 1, n85, p. 81-104.

DEDIEU J-P., 2011 L'internationalisme ouvrier à l'épreuve des migrations africaines en France.Critique internationale, $1, \mathrm{n}^{\circ} 50$, p. 145-167.

DRAHY J., 2004 Le droit contre l'État ? Droit et défense associative des étrangers : l'exemple de la Cimade. Paris : L'Harmattan.

DUDOUET F-X., GRÉMONT É., 2007 Les grands patrons et l'État français. 1981-2007.Sociétés contemporaines, vol.4, $\mathrm{n}^{\circ} 68$, p. 105-131.

DURIEZ H., 2004 Modèles d'engagement et logique de structuration des réseaux locaux de la gauche mouvementiste à Lille. Politix, vol. 17, nº8, p. 165-199.

ENGRAND S., 1973 L'alphabétisation bénévole des travailleurs immigrés. Esquisse d'une pratique du bénévolat, mémoire de maîtrise de sociologie, Univ. Lille 1.

FILLIEULE O., BLANCHARD P., AGRIKOLIANSKY E., BANDLER M., PASSY F., SOMMIER I., 2004. L'altermondialisation en réseaux. Trajectoires militantes, multipositionnalité et formes de l'engagement: les participants du contre-sommet du G8 d'Evian. Politix. Vol.17, nº68, p. 13-48.

FINEZ J., COMET C., 2011 Solidarités patronales et formation des interlocks entre les principaux administrateurs du CAC40. Terrains\&travaux, vol.2, n¹9, p. 57-76. 
FISCHER N., 2009a Jeux de regards. Surveillance disciplinaire et contrôle associatif dans les centres de rétention administrative. Genèses, $n^{\circ} 75$, p. 45-65.

FISCHER N., 2009b Une frontière « négociée ». L’assistance juridique associative aux étrangers placés en rétention administrative. Politix, $\mathrm{n}^{\circ} 87$, p. 71-92.

GALANO M., SPIRE A., 2002 «France confection » : le Sentier (1980). Plein Droit, n55.

GAXIE D., 1977 Économie des partis et rétributions du militantisme. Revue française de science politique, vol. $27, \mathrm{n}^{\circ} 1$, p. 123-154.

HAVARD DUCLOS B., 2002 Entre philanthropie et syndicalisme : militants et mal-logés de l'association «Droit au logement» (DAL), thèse de doctorat en sociologie, Univ. Versailles-Saint-Quentin-enYvelines.

HAVARD DUCLOS B., NICOURD S., 2005 Pourquoi s'engager? Bénévoles et militants dans les associations de solidarité. Paris : Payot.

HMED C., 2007 Contester une institution dans le cas d'une mobilisation improbable : la « grève des loyers » dans les foyers SONACOTRA dans les années 1970. Sociétés Contemporaines, n65, p. 55-81.

Insee, Enquête emploi, 2009.

Insee, Enquête SRCV-SILC, 2010.

Insee-DGI, Enquête Revenus fiscaux, 2005.

ISRAËL L., 2003 Faire émerger le droit des étrangers en le contestant ou l'histoire paradoxale des premières années du GISTI. Politix, vol. 16, nº62, p. 115-143.

ISRAËL L., 2009 L’arme du droit, Paris, Presses de Sciences Po.

LAURENS S., 2008 « 1974 » et la fermeture des frontières. Politix, nº2, p. 69-94.

LECHIEN M-H., 2002 Pratiques humanistes, engagements militants et investissements professionnels. Trois études de cas, thèse de doctorat de sociologie, EHESS.

LECHIEN M-H., 2003 Des militants de la « cause immigrée ». Genèses, vol. 1, n50, p. 91-110.

LENOIR R., 1989 Objet sociologique et problème social. CHAMPAGNE P., LENOIR R. , MERLLIÉ D., PINTO L., (dir.) Initiation à la pratique sociologique. Paris : Dunod, p. 65-77.

MAREK A., 2003L'usage militant de l'expertise juridique : le GISTI. LOCHARD Y., SIMONETCUSSET M., (dir.) L’expert associatif, le savant et le politique. Paris : Éditions Syllepse, p. 67-87.

MATHIEU L., 2006 La double peine. Histoire d'une lutte inachevée. Paris : La Dispute.

MATHIEU L., 2007 L’espace des mouvements sociaux. Politix, vol. 1, nº77, p. 131-151.

MATHIEU L., 2009 Les années 70, un âge d’or des luttes. Paris : Éditions Textuel. 
MATHIEU L., 2010a Soutenir les familles sans-papiers. L'engagement dans le Réseau éducation sans frontières. FASSIN D. (dir.) Les nouvelles frontières de la société française. Paris : La Découverte, p. 317337.

MATHIEU L., 2010b Les ressorts sociaux de l'indignation militante. L'engagement au sein d'un collectif départemental du Réseau éducation sans frontière. Sociologie, vol. 1, n³, p. 303-318.

MATHIEU L., 2012 L’espace des mouvements sociaux. Paris : Éditions du Croquant.

McADAM Doug (2012 [1988]), Freedom Summer. Luttes pour les droits civiques Mississippi 1964, Paris, Agone. Traduit de l'anglais par Célia Izoard.

MIZRUCHI M. S., 1996 What do interlocks do? An analysis, Critique, and Assessment of Research on Interlocking Directorates. Annual Review of Sociology, vol.22, n¹, p. 271-298.

MOUCHARD D., 2002 Les « sans-emploi.CRETTIEZ X., SOMMIER I. (dir.) La France rebelle. Tous les foyers, mouvements et acteurs de la contestation. Paris : Michalon.

OBERSCHALL A., 1973 Social Conflict and Social Movements. Englewood Cliffs: Prentice-Hall.

PAGIS J., 2011 Incidences biographiques du militantisme en Mai 68. Sociétés contemporaines, vol.4, n०84, p. 25-51.

PASSY F., 1998 L'action altruiste. Contraintes et opportunités de l'engagement dans les mouvements sociaux. Genève : Droz.

PÉCHU C., 2006 Droit Au Logement, genèse et sociologie d'une mobilisation. Paris : Dalloz.

PELLETIER D.,, SCHLEGEL J-L., (dir.) 2012 À la gauche du Christ. Les chrétiens de gauche en France de 1945 à nos jours. Paris : Seuil

PETTE M., 2012 S'engager pour les étrangers. Les associations et les militants de la cause des étrangers dans le Nord de la France. Thèse de doctorat de sociologie soutenue le 13 novembre 2012.

PETTE M., 2014 Associations : les nouveaux guichets de l'immigration? Du travail militant en préfecture. Sociologie, vol.5, n 4 , décembre 2014 (à paraître).

PITTI L., 2001 Grèves ouvrières versus luttes de l'immigration: une controverse entre historiens. Ethnologie française, vol.31, n³, p. 465-476.

REYNAUD E., 1980 Le militantisme moral. MENDRAS H., (éd.) La sagesse et le désordre. Paris : Gallimard.

ROUSSEAU S., 2008 Les cathos de gauche : l'engagement dans les luttes politiques. ARTIÈRES P., ZANCARINI-FOURNEL M. (dir.) 68 : une histoire collective, 1962-1981. Paris : La Découverte, p. 634644.

SIMÉANT J., 1998a La cause des sans-papiers. Paris : Presses de Sciences Po.

SIMÉANT J., 1998b L'efficacité des corps souffrants : le recours aux grèves de la faim en France. Sociétés Contemporaines, ${ }^{\circ} 31$, p. 59-86. 
SIMÉANT J., 2009 La grève de la faim. Paris : Presses de Sciences Po.

SOMMIER I., 2002 À la gauche de la gauche. CRETTIEZ X., SOMMIER I., (dir.) La France rebelle. Tous les foyers, mouvements et acteurs de la contestation. Paris : Michalon.

TILLY C., 1984 Les origines du répertoire d'action collective contemporaine en France et en GrandeBretagne. Vingtième siècle. Revue d'histoire, nº4, p. 89-108. 